\title{
Solitons in Nonlinear Media with an Infinite Range of Nonlocality: First Observation of Coherent Elliptic Solitons and of Vortex-Ring Solitons
}

\author{
Carmel Rotschild, Oren Cohen, Ofer Manela, and Mordechai Segev \\ Physics Department and Solid State Institute, Technion, Haifa 32000, Israel \\ Tal Carmon \\ California Institute of Technology, Pasadena, California 91125, USA \\ (Received 28 December 2004; published 17 November 2005)
}

\begin{abstract}
We present an experimental study on wave propagation in highly nonlocal optically nonlinear media, for which far-away boundary conditions significantly affect the evolution of localized beams. As an example, we set the boundary conditions to be anisotropic and demonstrate the first experimental observation of coherent elliptic solitons. Furthermore, exploiting the natural ability of such nonlinearities to eliminate azimuthal instabilities, we perform the first observation of stable vortex-ring solitons. These features of highly nonlocal nonlinearities affected by far-away boundary conditions open new directions in nonlinear science by facilitating remote control over soliton propagation.
\end{abstract}

DOI: 10.1103/PhysRevLett.95.213904

Nonlocality plays an important role in many areas of nonlinear physics. Nonlocality typically arises from an underlying transport mechanism (heat [1], atoms in a gas [2], charge carriers [3,4], etc.) or from long-range forces (e.g., electrostatic interactions in liquid crystals [5]) and many-body interactions as with matter waves in BoseEinstein condensates [6] or plasma waves [7]. In nonlinear optics specifically, nonlocality was found in photorefractives [3,8-10], in thermal nonlinear media [11-14], in atomic vapors [2], and in liquid crystals [5,15]. In principle, nonlocality acts to spread out the effects of localized excitations, and as such it can suppress modulation instabilities of homogeneous states [16]. However, in spite of the natural "averaging" tendency inherent to nonlocality, even highly nonlocal nonlinear media can support solitons [2,5,17-20]. Moreover, it was suggested that nonlocality can prevent the catastrophic collapse of self-focused beams, allowing $(2+1) \mathrm{D}$ solitons in Kerr-type media $[2,17,21]$. In a similar vein, it was proposed that nonlocality can suppress azimuthal instabilities of vortex-ring beams $[22,23]$, but such an experiment has thus far never been reported. Finally, nonlocality can considerably alter soliton interactions, e.g., giving rise to attraction between out of phase solitons $[19,20,24]$ and between dark solitons [25], which without nonlocality always repel, and causing attraction between well separated solitons [26].

Here we present an experimental study on solitons in a nonlinear medium with an extremely large range of nonlocality, such that far-away boundary conditions directly affect the soliton beam. We use the thermal optical nonlinearity in lead glass, which is of the self-focusing type. The nonlocal nature of this thermal nonlinearity is manifested in the heat-transfer (Poisson-type) equation, for which boundary conditions greatly influence the temperature distribution. The nonlinear index change is proportional to the temperature change; hence, the boundary conditions, even from afar, significantly affect the refrac-
PACS numbers: 42.65.Tg, 42.65.Jx, 47.27.Te

tive index structure supporting solitons. We show that setting transversely anisotropic boundary conditions (e.g., rectangular boundaries in the transverse plane) facilitates coherent elliptic solitons. We emphasize that coherent elliptic solitons cannot exist in local, spatially isotropic, nonlinear media, because such a nonlinearity cannot counteract the anisotropic diffraction of an elliptic beam [27]. Thus, our observation constitutes the first experimental observation of coherent elliptic solitons [28]. Subsequently, we exploit the natural tendency of long-range nonlocal nonlinearities to eliminate azimuthal instabilities and demonstrate the first experimental observation of stable vortexring solitons. This is in sharp contrast to the propagation of such beams in local nonlinear media [29], where azimuthal instabilities rapidly break up the ring beam [30]. Our observations demonstrate that extremely long-range nonlocal nonlinear response and support new kinds of solitons that have never been observed before, offering remote control over the propagation of solitons through boundary conditions.

Our experiments are carried out in lead glass exhibiting a self-focusing thermal nonlinearity: An increase in the optical intensity results in a nonlocal temperature increase and, consequently, to an increase in refractive index. Selftrapping of bell-shaped (circular) beams in such materials has been demonstrated decades ago [12]; nevertheless, the huge range of nonlocality has never been utilized to observe new kinds of solitons in this medium. In such media, the refractive index change increases linearly with temperature change [14] $\Delta n=\beta \Delta T, \beta=d n / d T$ is the thermal coefficient of the refractive index, and $\Delta n$ and $\Delta T$ are the index and temperature changes, respectively. The light beam gets slightly absorbed and heats the glass, serving as a heat source. The heat diffuses with a thermal conductivity, subject to the boundary conditions and the light beam that has induced it. The steady state temperature distribution $\Delta T$ establishes an index change $\Delta n$, which, under 
appropriate conditions, can support a spatial soliton. The formation of $\Delta n$ is a fully reversible process that does not involve any permanent material changes. In our lead glass samples, the refractive index is $n_{0}=1.8, \beta=$ $14 \times 10^{-6}\left[\mathrm{~K}^{-1}\right]$, the absorption coefficient is $\alpha \approx$ $0.01\left[\mathrm{~cm}^{-1}\right]$, and $\kappa=0.7[\mathrm{~W} /(\mathrm{m} \mathrm{K})]$. Even though such an absorption coefficient is very small (absorption can be neglected for distances up to $20 \mathrm{~cm}$ ), it is sufficient to support $50 \mu \mathrm{m}$ wide solitons, which, with a total power of $\sim 1 \mathrm{~W}$, give rise to $\Delta n \sim 5 \times 10^{-5}$. For our experiments with elliptic soliton, we use a series of samples of different lengths (in the propagation direction) of 17, 33, and $50 \mathrm{~mm}$, each cut to a rectangular cross section of $1 \times$ $2.4 \mathrm{~mm}$ (aspect ratio of 2.4), with the rectangular boundaries connected thermally to a heat sink at a fixed temperature. We use a $488 \mathrm{~nm}$ laser beam passed through two cylindrical lenses, perpendicular to one another, forming an elliptic beam at the input face of the sample (Fig. 1). We monitor the intensity distribution at the input and output faces by imaging the input and output beams onto a CCD camera. Figure 2 shows experimental results demonstrating elliptic solitons at different propagation distances: $50 \mathrm{~mm}$ [Figs. 2(a)-2(c)], $33 \mathrm{~mm}$ [Figs. 2(d)-2(f)], and $17 \mathrm{~mm}$ [Figs. 2(g) $-2(\mathrm{i})]$. The $50 \times 80 \mu \mathrm{m}$ FWHM input beams shown in the left column [Figs. 2(a), 2(d), and 2(g)] diffract at low power to the output shown in the center column [Figs. 2(b), 2(e), and 2(h)]. Note the transversely anisotropic diffraction of the elliptic beams; for example, after $50 \mathrm{~mm}$ propagation, the $50 \mu \mathrm{m}$ width diffracts to $130 \mu \mathrm{m}$, whereas the $80 \mu \mathrm{m}$ width broadens to $110 \mu \mathrm{m}$ [Fig. 2(b)]. Finally, the high power (1 Watt) output soliton beams are depicted in the right column [Figs. 2(c), 2(f), and 2(i)]. The top row presents the first experimental demonstration of the coherent elliptic soliton in isotropic nonlinear media [31]. As proven by Fig. 2, the self-trapped beam maintains its narrow elliptic profile at all propagation distances.

We emphasize that the thermal nonlinearity in lead glass does not have any intrinsic anisotropy, and the transverse symmetry is broken solely by the anisotropic boundary conditions. To prove this issue, we carry out experiments with a sample of a square cross section and confirm that symmetric boundary conditions support circular solitons but not elliptic solitons. Evidently, the formation of the elliptic solitons in Fig. 2 is enabled by the rectangular boundaries. To further confirm that indeed the symmetry

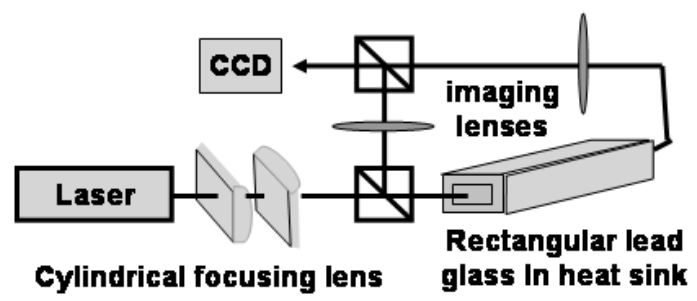

FIG. 1. Setup for the elliptic soliton experiments. of the soliton is determined solely by the (far-away) boundary conditions, we launch a circular beam into samples of rectangular cross sections at two orthogonal settings [Figs. 3(a) and 3(c)]. At low intensity, the beams diffract and the output beams are circular as expected (not shown here). But at high intensity, the initially circular beams reshape, self-focus, and become elliptic [Figs. 3(b) and 3(d)], with their major and minor axes corresponding to the longer and shorter distances from the boundaries, respectively. The nonlinear response follows the directionality of the far-away boundaries: The nearer transverse boundary enforced a larger temperature gradient, and thus a larger gradient of $\Delta n$, than the farther away boundary does. In this way, the elliptic beam is able to induce a stationary elliptic index change supporting an elliptic soliton. To complete our test-case studies, we launch a high intensity elliptic beam with the "wrong" orientation: the major axis of the ellipse parallel to the narrower direction of the sample [Fig. 3(e)]. The input elliptic beam reshapes and rotates its directionality to conform the elongated geometry of the sample [Fig. 3(f)], emerging as a selftrapped elliptic beam with its major axis in the direction of the farther away boundary. Thus, we have shown conclusively that the elliptic solitons in Fig. 2 are a direct consequence of transversely anisotropic boundary conditions, which determine the ellipticity of the solitons from far away. The elliptic solitons experiments demonstrate: (i) The nonlinear index change extends over a very large region beyond the extent of the optical beam. (ii) Far-away boundary conditions significantly affect the self-trapping, controlling a soliton beam "by remote."

As discussed above, it has been proposed [22,23] that nonlocal nonlinearities suppress azimuthal instabilities of vortex-ring beams, but experimental observation of stable vortex-ring solitons has thus far not been reported. Exploiting this stabilizing feature, we demonstrate the first stable vortex-ring solitons. In this experiment we use a square sample with a fixed temperature at its boundaries [32]. The dimensions of our sample are $2 \times 2 \times 28 \mathrm{~mm}$ (the latter being the propagation distance). As an input beam, we use the reflection from a unity-charge vortex

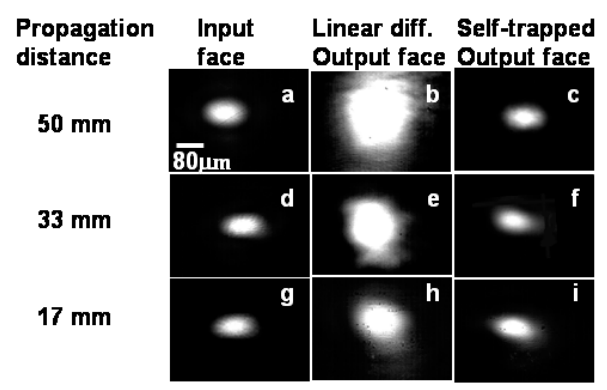

FIG. 2. Experimental results demonstrating elliptic solitons at different propagation distances: $50 \mathrm{~mm}$ (a)-(c), $33 \mathrm{~mm}$ (d)-(f), and $17 \mathrm{~mm}(\mathrm{~g})-(\mathrm{i})$, showing the input beam (left column), the diffracted output beam at low (center column), and the output soliton beam at high power (right column). 


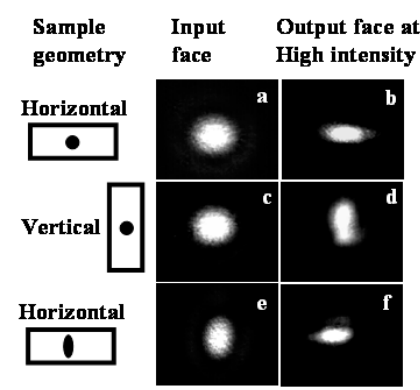

FIG. 3. Experiments demonstrating the evolution of a high intensity circular input beam (a),(c) into an elliptic beam (b),(d) under different boundary conditions (right column), and the evolution of a high intensity elliptic beam launched in the wrong direction (e),(f).

phase mask. Figure 4 shows a $50 \mu \mathrm{m}$ diameter (peak to peak) vortex-ring soliton. The input beam of Fig. 4(a) diffracts at low power $(10 \mathrm{mWatt})$ to the $120 \mu \mathrm{m}$ output beam of Fig. 4(b) after $28 \mathrm{~mm}$ propagation. At high power (1 Watt), the beam self-traps, forming a $56 \mu \mathrm{m}$ diameter vortex-ring soliton [Fig. 4(c)]. We verify that the soliton possesses a vortex phase structure by interfering the output beam with a spherical wave [Fig. 4(d)].

We now study theoretically the vortex-ring and the elliptic solitons within the model for thermal self-focusing nonlinearity [14]. The slightly absorbed light acts as a heat source, and the induced temperature change $\Delta T$ satisfies the heat equation in temporal steady state $[1,14]$

$$
\kappa \nabla^{2} T(x, y, z)=-\alpha I(x, y, z),
$$

where $I$ is the beam intensity distribution, and $\Delta n(x, y, z)$ is proportional to the local change in temperature $\Delta n=$ $\beta \Delta T$, with a coefficient $\beta$. The boundary conditions of Eq. (1) directly affect the temperature distribution and, hence, affect $\Delta n$ induced by $I$. Denoting the optical field as $E=A(x, y, z) e^{i(\omega t-k z)}+$ c.c., $A$ being the slowly varying amplitude of the beam, $k=\omega n_{0} / c, \omega$ the frequency, $n_{0}$ the unperturbed refractive index $\left(|\Delta n| \ll n_{0}\right), c$ the vacuum light speed, and $I=|A|^{2}$. The paraxial nonlinear wave equation is

$$
\nabla_{\perp}^{2} A+2 i k \frac{\partial A}{\partial z}+2 k^{2} \frac{\Delta n}{n_{0}} A=0 .
$$

We seek solutions of the form $A(x, y, z)=u(x, y) e^{i \gamma z}$, for

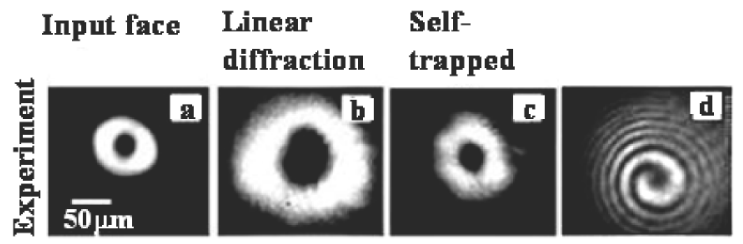

FIG. 4. Experimental results showing (a) input vortex beam, (b) diffracted output beam at low power after $28 \mathrm{~mm}$ propagation, (c) output soliton beam at high power, and (d) the vortex phase structure of the output soliton. which $I$ and $\Delta T$ are $z$-independent; hence, so is $\Delta n(x, y)$. We find solitons by solving Eqs. (1) and (2) selfconsistently, with boundary conditions such that $I$ vanishes at the boundaries and $\Delta T$ depending on the specific configuration. After finding the solitons, we test their stability in the presence of noise with a beam propagation code. We find both the elliptic and the vortex-ring solitons to be stable for more than ten diffraction lengths (limited by computation time only) against random noise (at $z=0$ ) in excess of $5 \%$ of the soliton wave function.

We first discuss bright vortex-ring soliton solutions under our experimental parameters. Figures 5(a)-5(c) show the calculated results depicting a $70 \mu \mathrm{m}$ diameter vortex-ring soliton. The input vortex-ring beam shown in Fig. 5(a) diffracts linearly at low power $(10 \mathrm{mWatt})$ to the $118 \mu \mathrm{m}$ output beam depicted in Fig. 5(b) after $28 \mathrm{~mm}$ propagation. At high power ( 1 Watt), the beam selftraps and forms a $70 \mu \mathrm{m}$ diameter vortex-ring soliton [Fig. 5(c)]. Figure 5(d) shows the transverse cross sections of the calculated vortex beam intensity and of $\Delta n(x, y)$, taken through the dark vortex center. The structure of $\Delta n(x, y)$ has a single peak: a global maximum at the vortex center, where the light is zero. This feature exemplifies the highly nonlocal nature of the nonlinearity: $\Delta n$ is maximal where the intensity is zero. This stands in sharp contrast to $\Delta n(x, y)$ induced by a ring beam in any local self-focusing media, where the beam forms a ring shape $\Delta n(x, y)$ with a minimum at the center, a structure which is azimuthally unstable. Comparing our experimental and calculated results, we find that the calculated soliton is $\sim 30 \%$ wider (under the same experimental parameters). Likewise, the experimental value at the center is $\sim 5 \times 10^{-5}$, whereas the calculated value is $2 \times 10^{-5}$.

Consider now the calculated elliptic solitons, which we find by solving Eqs. (1) and (2) self-consistently, with fixed temperature and vanishing intensity at the rectangular boundaries. We find that, indeed, when the shape of the bulk lead glass is rectangular, the temperature distribution is elliptic and supports elliptic solitons. However, according to the model, an elliptic soliton similar to the one we demonstrated experimentally $(\mathrm{FWHM}=50 \times 80 \mu \mathrm{m}$, $P=1 \mathrm{~W}$ ) requires a sample with much smaller cross sections. In addition, the calculated value of $\Delta n$ at the center of the ellipse is again smaller than the measured value. To verify that the nonlinearity in our experiment is indeed higher and more anisotropic than the calculated

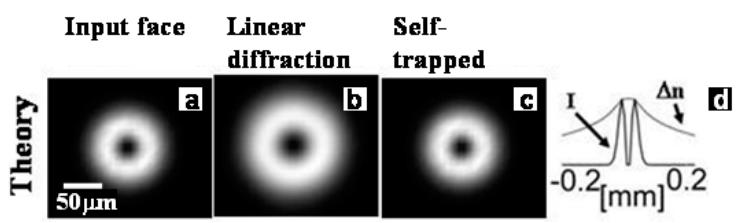

FIG. 5. Calculated results showing (a) input vortex beam, (b) diffracted output beam at low power after $28 \mathrm{~mm}$ propagation, (c) output soliton at high power, and (d) cross sections of $\Delta n$ and the intensity inducing it. 
thermal response, we repeat the elliptic soliton experiment and launch an additional (very weak) wave of a different wavelength, illuminating the sample uniformly and copropagating with the soliton, serving as a probe for the index structure induced by the soliton. We interfere the output wave with a plane wave and decipher $\Delta n(x, y)$. The measured value of $\Delta n$ at the center of the ellipse is $\sim 5 \times 10^{-5}$, whereas the calculated value is smaller, $\sim 2 \times 10^{-5}$. The measured eccentricity at the FWHM of the index change is $1: 2$, which occurs $\sim 140 \mu \mathrm{m}$ away from the soliton center. Closer to the boundaries (more than $300 \mu \mathrm{m}$ from the soliton center), the eccentricity exceeds 1:2.2, becoming more similar to the 1:2.4 ratio between the transverse cross sections of the sample. Evidently, our experimental results, which are fully reproducible with numerous samples and many experiments, cannot be explained quantitatively through the model represented by Eqs. (1) and (2). Thus, although qualitatively theory and experiments agree, our experiments suggest that there is another mechanism in our lead glass samples, which is not included in the model. That mechanism gives rise to an increased $\Delta n$ and is also significantly affected by the boundary conditions. A possible mechanism could be thermal stress, which typically involves birefringence. We test this issue experimentally and do not find evidence for any dependence of the soliton on the polarization of the light.

To conclude, we introduced a new feature of optically nonlinear response: a highly nonlocal nonlinearity that is significantly affected by far-away boundary conditions. In that sense, the geometry of the nonlinear medium, as well as the conditions at the boundaries (or elsewhere away from the beam), considerably affect the propagation of solitons and of other beams. We employed these features for the first observations of coherent elliptic solitons and vortex-ring solitons. These ideas of controlling highly localized beams from afar open new possibilities in soliton science, by offering remote control over soliton propagation. Similar behavior could be found in other systems, where the nonlinearity is highly nonlocal and the overall behavior is subject to boundary conditions. For example, such effects should exist with charge-density waves in relativistic plasma [33], as well as with gravitational selftrapping of photon beams [34]. In principle, such effects should exist whenever the nonlinearity is supported by a long-range transport or forces, subject, for example, to the Poisson equation. The challenge is to harness the longrange nonlocality to steer highly localized wave packets from far away, causing them to collide time and again in presubscribed fashion, forming networks of solitons.

This work was supported by the Israeli Science Foundation and the German-Israeli DIP Project.

[1] A. G. Litvak, JETP Lett. 4, 230 (1966).

[2] D. Suter and T. Blasberg, Phys. Rev. A 48, 4583 (1993).

[3] M. Segev et al., Phys. Rev. Lett. 68, 923 (1992).
[4] E. A. Ultanir et al., Opt. Lett. 29, 283 (2004); 28, 251 (2003).

[5] C. Conti, M. Peccianti, and G. Assanto, Phys. Rev. Lett. 91, 073901 (2003); 92, 113903 (2004); M. Peccianti et al., Nature (London) 432, 733 (2004).

[6] F. Dalfovo et al., Rev. Mod. Phys. 71, 463 (1999).

[7] H. L. Pecseli and J. J. Rasmussen, Plasma Phys. 22, 421 (1980).

[8] G. C. Duree et al., Phys. Rev. Lett. 71, 533 (1993).

[9] A. V. Mamaev et al., Phys. Rev. A 56, R1110 (1997).

[10] E. DelRe, A. Ciattoni, and A. J. Agranat, Opt. Lett. 26, 908 (2001).

[11] A. Dreischuh et al., Phys. Rev. E 60, 6111 (1999).

[12] F. W. Dabby and J. R. Whinnery, Appl. Phys. Lett. 13, 284 (1968).

[13] J. F. Henninot, M. Debailleul, and M. Warenghem, Mol. Cryst. Liq. Cryst. 375, 631 (2002).

[14] M. D. Iturbe-Castillo et al., Opt. Lett. 21, 1622 (1996).

[15] I. C. Khoo, Prog. Opt. 26, 107 (1988).

[16] A. G. Litvak et al., Fiz. Plazmy 1, 60 (1975).

[17] S. K. Turitsyn, Theor. Math. Phys. (Engl. Transl.) 64, 797 (1985).

[18] A. W. Snyder and D. J. Mitchell, Science 276, 1538 (1997).

[19] D. W. McLaughlin, D. J. Muraki, and M. J. Shelley, Physica (Amsterdam) 97D, 471 (1996).

[20] X. Hutsebaut et al., Opt. Commun. 233, 211 (2004).

[21] O. Bang et al., Phys. Rev. E 66, 046619 (2002).

[22] W. Krolikowski et al., J. Opt. B 6, S288 (2004).

[23] D. Briedis et al., Opt. Express 13, 435 (2005).

[24] I. A. Kolchugina, V. A. Mironov, and A. M. Sergeev, JETP Lett. 31, 304 (1980).

[25] N. I. Nikolov et al., Opt. Lett. 29, 286 (2004).

[26] M. Peccianti, K. A. Brzdkiewicz, and G. Assanto, Opt. Lett. 27, 1460 (2002).

[27] A. W. Snyder and D. J. Mitchell, Opt. Lett. 22, 16 (1997).

[28] We note that incoherent elliptic solitons are possible, by adjusting the transverse correlation function to obtain spatially isotropic diffraction, as suggested by E.D. Eugenieva, D. N. Christodoulides, and M. Segev, Opt. Lett. 25, 972 (2000) and demonstrated experimentally by O. Katz et al., Opt. Lett. 29, 1248 (2004).

[29] Note that it is possible to use spatial incoherence to suppress azimuthal instabilities upon a ring beam was suggested by C. Anastassiou et al., Opt. Lett. 26, 911 (2001) and demonstrated by C. C. Jeng et al., Phys. Rev. Lett. 92, 043904 (2004).

[30] Theory: V. I. Kruglov and R. A. Vlasov, Phys. Lett. 111A, 401 (1985); L. Torner and D. V. Petrov, Electron. Lett. 33, 608 (1997); W. J. Firth and D. V. Skryabin, Phys. Rev. Lett. 79, 2450 (1997). Experiments: V. Tikhonenko et al., Phys. Rev. Lett. 76, 2698 (1996); Z. Chen et al., Opt. Lett. 22, 1751 (1997).

[31] Elliptic solitons in transversely anisotropic nonlinear media are known to exist, e.g., in photorefractives at intensity ratios much smaller than unity. See A. A. Zazulya et al., Europhys. Lett. 36, 419 (1996).

[32] The small anisotropy introduced by the corners of a square sample are averaged out by the nonlocality, when the boundaries are far away from the localized beam.

[33] K. K. Mondal, A. R. Chowdhury, and S. N. Paul, Phys. Scr. 57, 652 (1998).

[34] L. A. Rivlin, Quantum Electron. 28, 99 (1998). 